


\title{
La regionalización de la educación superior en América Latina y el Sureste de Asia: experiencias contrastadas
}

\author{
PABlo Henri Ramírez
}

Realizó sus estudios de licenciatura en Periodismo y Comunicaciones en la Universidad Ming Chuan, gracias a una beca de estudios del gobierno de Taiwán, República de China. Obtuvo su maestría en Estudios Contemporáneos de Asia por la Universidad de Ámsterdam y su doctorado en Estudios Tailandeses por la Universidad de Chulalongkorn, gracias a una beca de investigación otorgada por la Embajada Real de Tailandia en México.

Desde el 2015 hasta finales de Julio de 2017, trabajó como Coordinador de Investigación en la Universidad de Bangkok, donde estuvo a cargo de la negociación de los acuerdos de colaboración y la movilidad académica con otras instituciones de educación superior, tanto de Asia como de Europa y América Latina. De agosto del 2017 a diciembre 2018 fue Coordinador de Internacionalización en la Unión de Universidades de América Latina y el Caribe, desarrolló estrategias de movilidad académica y docente, colaboración e investigación inter-institucional y el reconocimiento de créditos y títulos en la región de América Latina y el Caribe. En 2019 y principios de 2020 se desempeñó como asesor del Subsecretario de Educación Básica en la Secretaría de Educación Pública de México en donde participó en el diseño de las estrategias nacionales de educación inclusiva y de educación inicial. Desde abril del presente año es asesor político a cargo de los temas de educación y desarrollo en la embajada de Nueva Zelanda en México.

\section{Resumen}

Este artículo analiza y compara los procesos de regionalización de la educación superior en dos territorios opuestos del mundo. Por un lado el Sureste de Asia y por el otro América Latina y el Caribe. Ambas regiones han buscado crear su espacio común de educación superior desde la década de los años 90. Sin embargo, obtener el reconocimiento de títulos y créditos universitarios ha sido un camino tortuoso, a causa de las diferencias políticas y culturales entre los países de ambas regiones, así como por la falta de un interés verdadero por parte de las universidades y de los gobiernos locales para facilitar los procesos de reconocimiento.

Palabras clave:

Regionalización, Educación Superior, Sureste de Asia, América Latina, Política educativa. 


\title{
A regionalização da educação no nível superior da América Latina e o Suleste da Ásia: experiências contrastadas
}

\section{Sumário}

Esse artigo procura analisar e comparar os processos da regionalização da educação no nível superior em duas regiões contrárias no mundo. Por um lado o Suleste da Ásia e pelo outro a América Latina e o Caribe. As duas regiões tem procurado criar um espaço comum em educação superior desde a década dos anos 90, porém, obter o reconhecimiento dos títulos e créditos universitários tem sido um caminho torturante a causa das diferenças políticas e culturais entre os países das duas regiões, assim como também pela falta dum verdadeiro interesse por parte das universidades e dos governos locais por facilitar os processos de reconhecimiento.

Palavras-chave

Regionalização; Educação no nível superior; Suleste da Ásia; América Latina; Política educativa.

\section{The regionalisation of Higher Education in Latin America and South-East Asia: Contrasting Experiences}

\begin{abstract}
This study consists in analysing and comparing the processes of regionalisation of two opposite higher education areas of the world: on one side, South-East Asia; on the other side, Latin America and the Caribbean. Both regions have sought the creation of a common space of higher education since the '90s; nevertheless, obtaining the recognition of academic degrees and credits has been a difficult quest, due to the political and cultural differences between countries of the two areas, as well as the universities and local governments' lack of true interest to ease the processes of recognition.
\end{abstract}

\section{Key words}

Regionalisation; higher education; South-East Asia; Latinoamerica and the Caribbean; educative politics. 


\section{La regionalización de la educación superior}

En las últimas décadas, las políticas de educación superior han pasado de ser objeto reservado a los gobiernos nacionales a decisiones tomadas en pos del beneficio de una región u organización regional. En Europa, el Proceso de Bolonia ha sido fundamental para la creación del Espacio Europeo de Educación Superior lo que ha permitido la libre circulación de estudiantes, personal académico y la colaboración para la creación y diseminación del conocimiento a través de estructuras de títulos comunes y transferencia de créditos (Gornitzka, 2010; Ravinet, 2008). Sin embargo, esto no ha sido un ejercicio exclusivo de Europa, existen esfuerzos similares en la creación de estructuras de títulos comunes de educación superior en África (Hoosen, Butcher y Njenga, 2009), América Latina (Verger y Hermo, 2010) y Asia Oriental (Knight, 2012). Este fenómeno del "regionalismo de la educación superior", ha sido definido como "un proyecto político de creación de región que involucra al menos a una autoridad estatal, ya sea nacional, supranacional o internacional, quien a su vez designa y delimita la región geográfica del mundo a la que se extienden las políticas de educación superior creadas" (Chou y Ravinet, 2015: 368). Estas políticas de educación superior regionales ponen de lado la diversidad regional para enfocarse en la movilidad intrarregional y el reconocimiento de títulos para que las plantas docentes, los estudiantes y el conocimiento creado en las universidades puedan competir en el mercado mundial de la educación superior.

En este artículo, compararemos y analizaremos dos ejemplos de regionalismos de la educación superior para entender cómo surgen y se institucionalizan las ideas políticas del regionalismo de la educación superior en dos partes del mundo totalmente distintas. Para llevar a cabo este ejercicio, se seleccionaron el Sureste asiático y América Latina, dos regiones que han intentado llevar a cabo sus propios procesos de regionalización de la educación superior a través da lo que se ha llamado "la exportación del Proceso de Bolonia". Esta idea argumenta que los desarrollos llevados a cabo con el Proceso de Bolonia en Europa beneficiaron no solo al sector de la educación superior, si no que desencadenaron intereses políticos en otras áreas de la dimensión regional. Por lo tanto, el Proceso de Bolonia puede verse como un actor para la cooperación en políticas de educación superior para Europa (Huisman, Aldeman, Hsieh, Shams \& Wilkins, 2012). A pesar de que esta es una definición euro-centrista que ve a la difusión de las políticas de educación superior como unidireccional y todo poderosa es verdad que se han llevado a cabo procesos similares en otras partes del mundo y en las dos regiones que nos interesan. Sin embargo, es necesario tomar en cuenta que tanto en América Latina como en el Sureste de Asia, los desarrollos regionales se han llevado a cabo de manera endógena a cada región, tomando en cuenta las particularidades de cada una de ellas y sus formas de hacer las cosas. Para este trabajo se rastrearán y compararán los marcos de ideas de cada región para entender cómo surgen y evolucionan las ideas del regionalismo de la educación superior en América Latina y en el Sureste de Asia. Esto nos permitirá saber si la tesis sobre exportación del Proceso de Bolonia es una de las varias explicaciones posibles sobre el entusiasmo de crear espacios comunes de educación superior en otras partes del mundo fuera de Europa. 


\section{Creando el espacio común de educación superior en el Sureste asiático: la ASEAN Way educativa}

Desde el principio los gobiernos de las naciones del Sureste asiático ponen énfasis en que la cooperación política regional debe de ir acompañada por la cooperación en temas educativos. De hecho, poco después de que la Oficina de la UNESCO en Asia y el Pacífico fuese establecida en Bangkok en 1961, se creó la Organización de Ministerios de Educación del Sureste de Asia (SEAMEO por sus siglas en inglés) en 1965 para promover la cooperación regional en las áreas de educación, ciencia y cultura. De manera similar, poco después del lanzamiento de la Asociación de Estados del Sureste de Asia (ASEAN por sus siglas en inglés) en 1967, se crean las reuniones ministeriales de educación de la ASEAN (ASED por sus siglas en inglés). Sin embargo, este foro rápidamente quedó inactivo, dejando a SEAMEO la cooperación intergubernamental en cuestiones de política educativa hasta la fecha (Dang, 2012).

En este primer periodo de integración regional en el sureste asiático sobresalen dos características. Primero, la educación superior no se destacó como una de las prioridades de la política educativa dentro del esquema más amplio de la cooperación política, fue simplemente algo que se añadió de manera natural a los acuerdos. En segundo lugar, los esfuerzos para implementar un regionalismo educativo propio a la ASEAN se centraron en establecer primero las infraestructuras política necesarias para poder organizar a los diferentes estados y sus ministerios dejando en un segundo plano el contenido sustantivo de las políticas educativas regionales.

No es hasta principios de la década de 1990 que se nota un cambio notable enfocado en la conformación de un espacio común de educación superior en el sureste asiático. Durante esa década, dos organismos regionales jugaron un papel fundamental en la profundización de la cooperación educativa en la región aunque, cada uno de ellos representa una idea política diferente de cómo se entiende el regionalismo de la educación superior en esa parte del mundo. El primero de estos dos organismos es la Red de Universidades de la Asociación de Naciones del Sureste de Asia (AUN por sus siglas en inglés) que fue establecida en 1992 en Bangkok, Tailandia, y que tiene 
como misión coordinar las actividades en treinta instituciones emblemáticas de educación superior de la región. La AUN gestiona una variedad de redes de investigación y becas de movilidad entre las mejores universidades de cada país miembro de la ASEAN. Todas las instituciones miembros están invitadas a participar en los proyectos y deciden caso por caso si se involucran o no. Las actividades de AUN también incluyen a países no pertenecientes a la ASEAN como lo son Japón, China y Australia. Cabe mencionar que AUN no es un organismo independiente, sino uno de los muchos organismos sectoriales de los ministerios de educación de los miembros de la ASEAN. Sin embargo, la estructuración organizacional de AUN promueve una idea política específica de lo que la región espera de su regionalismo de educación superior: se trata de establecer redes específicas en cada disciplina entre los investigadores y alumnos de las mejores universidades de la región.

El segundo organismo regional responsable del desarrollo de un espacio común de educación superior en el sureste asiático es el Centro Regional de Educación Superior y Desarrollo de la SEAMEO (RIHED por sus siglas en inglés). La membresía actual de SEAMEO y ASEAN son las mismas, con la excepción de Timor Leste, que no es miembro de la ASEAN todavía. Al día de hoy, SEAMEO permanece fuera del marco institucional de la ASEAN. Desde que fue establecido por los ministros de SEAMEO en Bangkok en 1993, el RIHED ha trabajado en desarrollar 5 áreas objetivo, todas orientadas a fomentar el "acceso, la excelencia y la sinergia en la educación superior para el desarrollo regional". Originalmente creado y establecido en 1970 en Singapur, la idea de RIHED surgió de una colaboración conjunta entre la UNESCO, la Asociación Internacional de Universidades y la Fundación Ford. El RIHED trabaja una idea política diferente a la de la AUN ya que entiende el regionalismo de la educación superior del Sureste de Asia como una cooperación entre actores gubernamentales con poderes para decidir y adoptar políticas y objetivos sectoriales. Tanto AUN como RIHED han encabezado iniciativas y proyectos de educación superior que incluyen programas de movilidad estudiantil, reconocimiento de créditos y títulos, y, marcos de garantía de calidad tanto de universidades como de programas.

A partir de 2005, el regionalismo de la educación superior en el sureste asiático volvió a transformarse ya que los gobiernos de la región impulsaron activamente políticas de cooperación en sus políticas nacionales de educación superior. Este renovado interés por la cooperación regional nace de las cada vez mayores preocupaciones por la "dimensión externa" del Proceso de Bolonia (Zgaga, 2006), es decir, por el interés político en promover el Proceso de Bolonia como modelo de cooperación regional en políticas de educación superior en otras partes del mundo.

La singularidad de la cooperación regional en educación superior en la ASEAN puede ser explicada a través del legado histórico de la región. Desde la creación de los estados nación que conforman la ASEAN, la cooperación política se ha caracterizado por la informalidad, la no injerencia en los asuntos nacionales, la consulta sin confrontación y la creación de consenso como el principal modo de toma de decisiones, en lo que se conoce como la ASEAN Way (Koga, 2010). Este método de cooperación es único en la región y ha permeado a las políticas educativas, como se han visto en los casos de 
la AUN y en el RIHED, la cooperación en educación superior se lleva a cabo mediante la creación plataformas para intercambios constructivos entre sus miembros y generar consensos no vinculantes (Dosch, 2011). Esta manera de trabajar juntos es muy diferente a la forma en la que se llevó la integración europea en materia de educación superior. En Europa, de manera general, se establecieron instituciones supranacionales independientes y se adoptaron normas vinculantes en una variedad de sectores.

Este enfoque exclusivo a la ASEAN refleja la preferencia general de los estados miembros participantes para evitar cualquier posible combinación de poderes de toma de decisiones entre los funcionarios estatales y los representantes de la ASEAN. En este contexto, es sorprendente que a mediados de los años 2000, surgiera la idea y el acuerdo de crear un espacio común de educación superior en el sureste de Asia.

En 2007, la ASEAN anunció que el año 2015 sería la fecha límite para la creación de la Comunidad Económica de la ASEAN. Este compromiso político obligó a las otras dos áreas de cooperación fuera del ámbito económico de la ASEAN a avanzar en la cooperación de sus respectivos sectores. Estas áreas son la cooperación sociocultural y la cooperación en asuntos de seguridad. El área sociocultural decidió utilizar el discurso del conocimiento y su énfasis en el rol que juega la educación en el crecimiento económico, la competencia internacional y la cohesión social para avanzar en la creación del espacio común de educación superior de la ASEAN. Ya que dado el papel sociopolítico de la educación superior y su impacto socioeconómico en los países miembros, hubiese sido imposible no integrar a la educación en la idea de una comunidad económica en el sureste de Asia.

Este discurso del conocimiento marcó el comienzo de la posibilidad de establecer un espacio común de educación superior en la región. De igual forma, salieron a la luz los problemas que esto conllevaría para mantener la ASEAN Way en el ámbito educativo. La ASEAN le pidió al RIHED que realizara un ejercicio de mapeo de iniciativas regionales de educación superior que pudiesen proporcionar "un análisis sobre obstáculos, oportunidades y la relevancia de la ASEAN como una plataforma regional de cooperación e integración educativa" (Yavaprabhas, 2010: 10).

Presentado en 2008, este informe reveló que el Proceso de Bolonia fue solo una de varias iniciativas regionales bajo consideración de la Unión Europea para la creación del espacio común de educación superior europeo (SEAMEO RIHED, 2008). Además, el informe advirtió explícitamente que el enfoque general del proceso de Bolonia debía de evitarse debido a las "dudas y sospechas existentes entre los países del Sureste de Asia" (SEAMEO RIHED, 2008: 82).

Esto llevó a los estados miembros de la ASEAN a replantearse su idea del regionalismo de educación superior en el Sureste de Asia para incluir las normas específicas y la cultura de cada país a fin de lograr implementar una idea transnacional como lo es la creación de un espacio común de educación superior en la región (Jetschke, Acharya, De Lombaerde, Kastumata y Pempel, 2015 : 23). De esta manera, los instrumentos existentes como la AUN y el RIHED han usado el discurso del conocimiento como el escudo que les permite poner a la educación superior como motor económico y social 


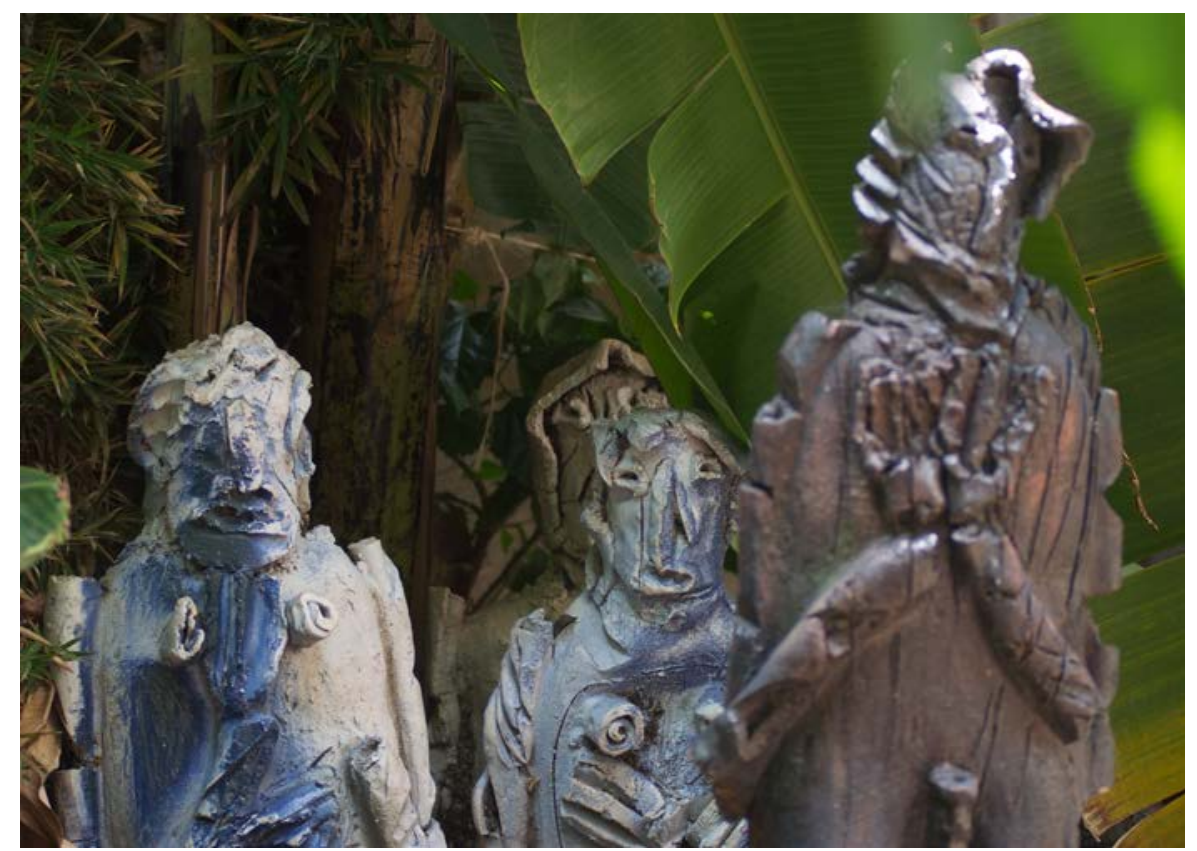

de la región guardando siempre las diferencias entre los estados y los múltiples actores involucrados.

Este reconocimiento de las diferencias de cada país, nos permite entender el espacio común de educación superior del Sureste de Asia como una multiplicidad de medidas y estructuras de gobernanza regionales en el ámbito de la política de educación superior de la región. Del mismo modo, la ASEAN Way inculcó los principios de no interferencia en los asuntos nacionales y la consulta sin confrontaciones, lo que finalmente condujo al establecimiento de 2 plataformas distintas para la cooperación en políticas de educación superior: el Centro Regional de Educación Superior y Desarrollo de la Asociación de Ministerios de Educación del Sureste de Asia (el RIHED) y la Red de Universidades de las Naciones del Sureste de Asia (la AUN) estas dos plataformas trabajan de manera paralela y enfocada a las necesidades individuales de sus miembros sin confrontarse los unos con los otros. Finalmente, el discurso del conocimiento, al destacar la importancia de los sectores de la educación para el avance colectivo de las sociedades y naciones, ayudó a que los estados de la región crearan el espacio común de educación superior en el Sureste de Asia. Este discurso del conocimiento fue el gancho que permitió que la cooperación en políticas de educación superior se hiciera factible en una región donde la no intervención era el método normativo de operación. Esto ha llevado a la creación de programas de movilidad y reconocimiento de títulos entre las mejores universidades de la región así como a la creación de marcos de calidad de programas e instituciones reconocidos por todos los países del Sureste Asiático. Ahora veamos como se ha llevado el proceso de regionalización de la educación superior en América Latina y el Caribe. 
ENLACES: la frustración latinoamericana y caribeña por crear un espacio común de educación superior en la región

En América Latina desde la Conferencia Regional de Educación Superior (CRES), de 1996, en la Habana, Cuba se empezaba a hablar de la necesidad de crear un espacio común de educación superior en la región. Sin embargo, no es hasta la CRES 2008 de Cartagena de Indias, Colombia, que se declara la intención de lograr una integración regional y la internacionalización de la educación superior a través de la consecución del Espacio de Encuentro Latinoamericano y Caribeño de Educación Superior (ENLACES).

De acuerdo con el Instituto Internacional de la UNESCO para la Educación Superior en América Latina y el Caribe (IESALC), ENLACES es 'una plataforma regional para la movilización y articulación de acciones concretas de cooperación académica solidaria que promuevan el conocimiento de las características, tendencias y problemas de la Educación Superior en la región; el diálogo entre los actores institucionales con miras a la consolidación de una agenda de consenso encaminadas a la superación de nuestras deficiencias y la promoción de una Educación Superior inclusiva, de calidad y pertinente; el apoyo a los procesos de reforma y/o fortalecimiento de los sistemas nacionales y de las instituciones de Educación Superior y la divulgación de informaciones vinculados a esos procesos' (IESALC-UNESCO, 2008).

El anuncio de la creación de ENLACES fue una de las resoluciones más importantes de la CRES 2008. Sin embargo, poco o nada se ha avanzado en torno a la creación de un verdadero espacio común de educación superior en América Latina y el Caribe. Uno de los factores importantes de este fracaso ha sido la falta de apoyos económicos por parte de los gobiernos y las universidades de la región. En efecto, este proyecto busca ser una plataforma de promoción de la "cooperación académica solidaria" sur-sur lo cual requiere de financiamiento importante por parte de todos los Estados para lograr una educación superior inclusiva, de calidad y pertinente así como poder ofrecer una oportunidad de movilidad estudiantil en la región más desigual del mundo. Desgraciadamente, las crisis económicas mundiales y locales que han azotado a la región desde 2007 han imposibilitado 
que los Estados inviertan de manera adecuada a la creación del ENLACES.

A pesar del fracaso que ha representado ENLACES desde 2008, existen intentos de conformar espacios comunes de educación superior regionales en América Latina y el Caribe y en este artículo nos centraremos en dos de ellos, el Sistema de Acreditación Regional de Carreras Universitarias de los Estados Partes del MERCosur y Estados Asociados y el Programa de Cooperación e Integración Universitaria de la UDUAL.

El Sistema de Acreditación Regional de Carreras Universitarias de los Estados Partes del MERCOSUR y Estados Asociados se creó en 1998 por los 4 países de MERCosur, (Argentina, Brasil, Paraguay y Uruguay), así como Bolivia y Chile como países asociados para lograr el reconocimiento de títulos universitarios emitidos en cada país en las áreas de agronomía, ingeniería y medicina. Estas carreras fueron escogidas por los países participantes para responder a las necesidades de los Estados de contar con profesionales formados en áreas económicas importantes para la región (Perotta, 2012).

El desarrollo de este proceso de acreditación tuvo muchas singularidades pues en lugar de procurarse una agencia acreditadora supranacional, cada país designó a una agencia local como su agencia nacional para que sea esta la que lleve los procesos de acreditación de las carreras en las universidades interesadas en participar. No obstante, los parámetros y procedimientos de la evaluación son comunes a todas las agencias acreditadoras y fueron aprobados por los ministros de educación de todos los países participantes (Ramírez Didou, 2019).

El éxito del programa ha sido importante, a los 6 países fundadores se les ha sumado Venezuela y, a nivel de programas, se han incluído las carreras de arquitectura, enfermería y odontología. Gracias a este sistema se han fortalecido los lazos entre aquellos encargados de los procesos de aseguramiento de la calidad en los países participantes y esto ha ayudado a que exista un mejor entendimiento de los procesos de evaluación de los programas educativos seleccionados. Sin embargo, este no es el único intento de crear un espacio común de educación superior en América Latina y el Caribe.

El 23 de enero 2018, en las instalaciones de la rectoría de Universidad Nacional Autónoma de México (UNAM), se firmó el Programa de Cooperación 
e Integración de las Universidades de América Latina y el Caribe (PCIU-UDUAL). Este programa busca el reconocimiento automático de créditos y títulos universitarios en las carreras de administración, enfermería, filosofía, física, ingeniería civil, matemáticas y química entre las universidades participantes. El grupo piloto de 10 universidades que firmaron este acuerdo son, además de la UNAM, la Universidad de Guadalajara (UDG), la Universidad de Panamá (UP), la Universidad Nacional de Avellaneda (UNDAV), la Universidad Nacional de San Juan (UNSJ), la Universidad Nacional de Colombia (UNAL), la Universidad Pontificia Católica del Perú (PUCP), la Universidad de la Habana (UH), la Universidad Estatal de Campinas (UNICAMP) y la Universidad Federal de Minas Gerais (UFMG).

Este acuerdo es un paso importante porque tiene un alcance regional de toda América Latina y el Caribe. Hasta este momento se han realizado a cabo reuniones de trabajo entre los decanos de cada carrera participante para decidir cómo se llevarán a cabo los procesos de movilidad de estudiantes y docentes. Sin embargo, es tiempo de que se saque la convocatoria para empezar a mover estudiantes entre las universidades participantes y ver qué influencia tendrá este programa en los estudios de posgrado de las generaciones futuras. También es necesario que se inviten a más universidades a participar en el programa e ir aumentando la selección de carreras participantes para, poco a poco, ir expandiendo este pequeño núcleo de espacio común de educación superior en la región. Sería interesante ver si este mismo programa puede ser aplicado a las carreras de las universidades tecnológicas de América Latina y el Caribe pues representan a un importante número de instituciones de educación superior y, por ende, de estudiantes y profesionales.

Desgraciadamente, a pesar de las buenas intenciones de las universidades creando este tipo de proyectos, no podemos dejar de lado que para mantenerlos, es necesario contar con importantes apoyos económicos y la financiación en educación superior no es una de las prioridades presupuestales de los gobiernos de la región. Cabe destacar sin embargo, que el Sistema de Acreditación Regional de Carreras Universitarias de los Estados Partes del MERcosur y Estados Asociados cuenta con el respaldo de los gobiernos participantes ya que consideran que las carreras que participan en el programa son de vital importancia para sus economías y, es probable, que para poder sobrevivir y expandirse el Programa de Cooperación e Integración de la UDUAL deba de buscar una alianza con los gobiernos nacionales de los países participantes para crear un fondo que permita la movilidad de estudiantes y docentes así como la cooperación y la investigación conjunta. 


\section{Conclusión}

Como se ha visto en este trabajo, tanto el Sureste de Asia como América Latina y el Caribe intentan crear sus espacios comunes propios en educación superior para garantizar una educación de calidad, inclusiva y que beneficie al desarrollo de sus regiones. Sin embargo, a pesar de las diferencias entre las regiones a nivel de tamaño, población, idiomas, sistemas educativos y culturas, ambas regiones pueden aprender de las experiencias de la otra a la hora de formar su espacio común de educación superior.

América Latina y el Caribe pueden analizar cómo funciona la ASEAN Way, esa manera de consultar sin confrontación y el consenso como el principal modo de toma de decisiones para así, sin vulnerar la autonomía universitaria, lograr avanzar en la inclusión de más universidades y carreras en sus programas de regionalización. Además, es posible conformar un organismo supranacional que, en conjunto con los ministerios de educación de la región y las universidades, siente las bases para la acreditación de programas y universidades latinoamericanas y caribeñas. La acreditación sería voluntaria por parte de las universidades y garantizaría el poder participar en los programas de regionalización establecidos.

La red de Universidades del Sureste de Asia y el Grupo de Ministerios de Educación del Sureste de Asia pueden aprender cómo se han creado acreditado los programas e instituciones en el contexto del Sistema de Acreditación Regional de Carreras Universitarias de los Estados Partes del MERCOSUR pues sienta bases comunes dejando la acreditación a las acreditadoras locales, sin meterse en los asuntos internos de cada país e institución.

Así, dos regiones opuestas geográficamente tienen problemas y necesidades similares. La cooperación entre ellas debería de ser mas cercana ya que tenemos más en común que de diferente.

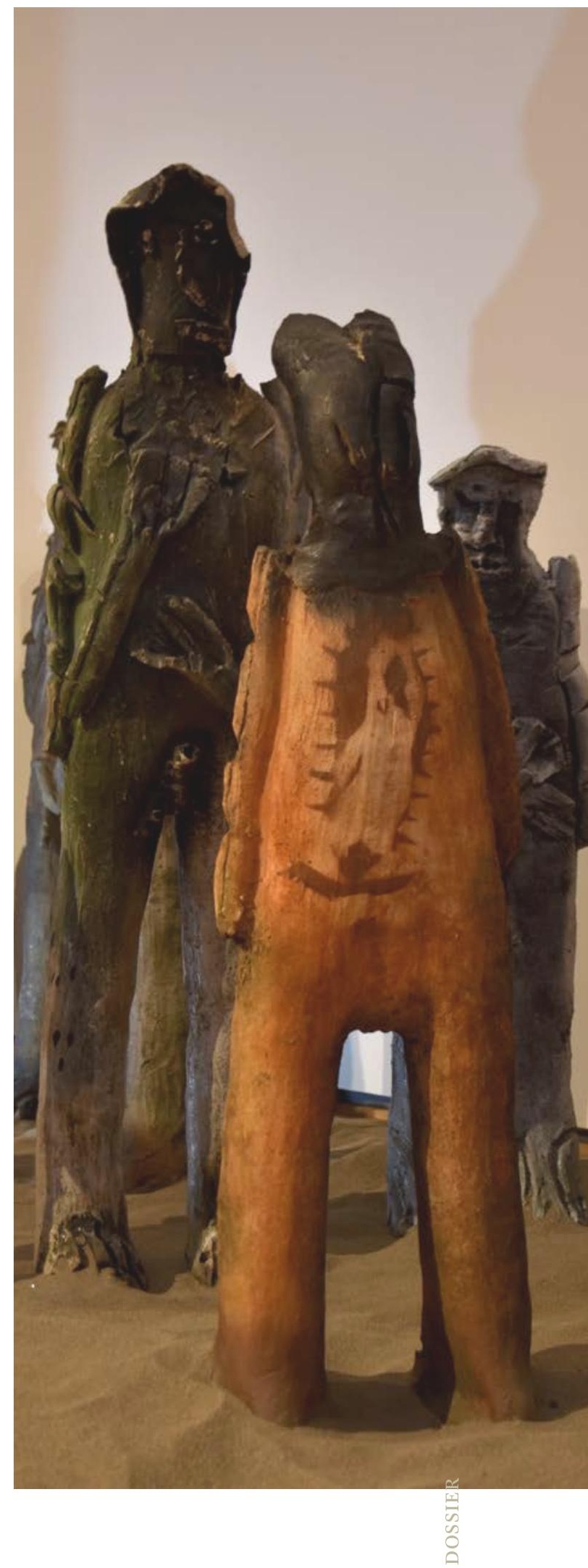

Universidades $\mid$ núm. 84, abril-junio 2020 | UDUAL | DOI:https://doi.org/10.36888/udual.universidades.2020.84.101 La regionalización de la Educación Superior en América Latina y el Sureste de Asia: experiencias contrastadas $\mid$ Pablo Henri Ramírez 21 


\section{Referencias}

Chou, M.-H., \& Ravinet, P. (2015). The rise of "higher education regionalism": An agenda for higher education research. In H. De Boer, D. Dill, J. Huisman, \& M. Souto-Otero (Eds.), Handbook of higher education policy and governance (pp. 361-378). Palgrave: Basingstoke.

Dang, Q. A. (2012). The Bologna process goes east? From "third countries" to prioritizing interregional cooperation between the ASEAN and EU. In A. Curaj, P. Scott, L. Vlasceanu, \& L. Wilson (Eds.), European higher education at the crossroads: Between the bologna process and national reforms (pp. 763-783). Dordrecht: Springer.

Dosch, J. (2011). Southeast Asia: ASEAN and the challenge of regionalism. In M. K. Connors, R. Davidson, \& J. Dosch (Eds.), The new global politics of the Asia Pacific (2nd ed., pp. 121-139). London: Routledge.

Gornitzka, Å. (2010). Bologna in context: A horizontal perspective on the dynamics of governance sites for a Europe of knowledge. European Journal of Education, 45, 536-548.

Hoosen, S., Butcher, N., \& Njenga, N. K. (2009). Harmonization of higher education programmes: A strategy for the African Union. African Integration Review, 3(1), 1-36.

Huisman, J., Adelman, C., Hsieh, C.-C., Shams, F., \& Wilkins, S. (2012). Europe's Bologna process and its impact on global higher education. In D. K. Deardorff, H. de Wit, J. Heyl, \& T. Adams (Eds.), The SAGE handbook of international higher education (pp. 81-100). Thousand Oaks, CA: Sage.

IESALC-UNESCO (2008). CRES 2008. Recuperado de www.unesco.org.ve/index.php? option=com_content\&view=article\&id=365\&Itemid=423\&lang=pt

Jetschke, A., Acharya, A., De Lombaerde, P., Katsumata, H., \& Pempel, T. J. (2015, April). Roundtable: Studying Asian and comparative regionalism through Amitav Acharya's work. International Relations of the Asia-Pacific, 26, 1-30.

Knight, J. (2012). A conceptual framework for the regionalization of higher education: Application to Asia. In J. N. Hawkins, K. H. Mok, \& D. E. Neubauer (Eds.), Higher education regionalization in Asia pacific (pp. 17-35). New York, NY: Palgrave Macmillan.

Ravinet, P. (2008). From voluntary participation to monitored coordination: Why European countries feel increasingly bound by their commitment to the Bologna process. European Journal of Education, 43, 353-367.

Koga, K. (2010). The normative power of the "ASEAN way": Potentials, limitations and implications for east Asian regionalism. SJEEA, 2010, 80-95. Winter.

Perotta, D. (2012), La integración regional de la educación superior en el MERCOSUR en el marco de la orientación general del bloque y la tensión entre un modelo solidario y otro competitivo: el caso de los programas regionales de acreditación de carreras de grado (del MEXA al ARCU-SUR, 1998-2008), Tesis para la obtención del grado de magister, FLACSO Argentina.

Ramirez Didou, P. (2019) Acreditación internacional de la calidad en la educación superior: Pendientes y proyectos regionales en América Latina', en: Comas, Javier: La Internacionalización de la Educacion Superior una apuesta y una oportunidad del presente, Colección Documentos ANUIES, Universidad Autónoma de México.

SEAMEO RIHED. (2008). Harmonisation of higher education: Lessons learned from the Bologna process. Bangkok: Author.

Verger, A., \& Hermo, J. P. (2010). The governance of higher education regionalisation: Comparative analysis of the Bologna process and MERCOSUR-Educativo. Globalisation, Societies and Education, 8, 105-120.

Yavaprabhas, S. (2010, May 10-11). SEAMEO RIHED and the progress towards a common space of higher education in Southeast Asia. Paper presented at the Regional Workshop on Educational Cooperation in East Asia Summit Countries, Jakarta, Indonesia.

Zgaga, P. (2006). Looking out: The Bologna process in a global setting. On the "external dimension" of the Bologna process. Oslo: Norwegian Ministry of Education and Research. 